\title{
Evaluación neuropsicológica en 129 pacientes chilenos con esclerosis múltiple recurrente remitente previo a inicio de fármacos inmunomoduladores
}

\author{
JORGE NOGALES-GAETE ${ }^{1,2}$, RODRIGO ARACENA ${ }^{1,2}$, VANNIA DÍAZ ${ }^{1}$, \\ PEDRO ZITKO $^{3}$, CLAUDIO ELOIZA ${ }^{1}$, SERGIO CEPEDA-ZUMAETA ${ }^{1}$, \\ PAULA AGURTO $^{1}$, CAROLA GONZÁLEZ $^{1}$
}

\section{Neuropsychological assessment of patients with relapsing remitting multiple sclerosis prior to the use of immunomodulatory drugs}

Background: The detection of cognitive changes (CC) and psychiatric disorders in relapsing remitting multiple sclerosis (MS-RR) contributes to patient clinical monitoring. Aim: To assess the frequency and characteristics of CC and psychiatric disorders in Chilean patients with MS-RR, before starting immunomodulatory treatment. Patients and Methods: Retrospective review of data that was obtained following a standard assessment protocol. It consisted in the application of the Expanded Disability Status Scale of Kurtzke (EDSS), Multiple Sclerosis Functional Composite (MSFC), fatigue intensity scale of Krupp, brief repeatable battery of neuropsychological Rao (BRN-R) and Hamilton's depression and anxiety questionnaires. Results: We evaluated 129 patients aged between 12 and 60 years of age (69\% women). Ninety four percent of patients had eight or more years of schooling. The average EDSS score was 2.83. CC were detected in $62 \%$ of participants, in at least one subtest of the BRN-R. The main changes were verbal memory and speed in the processing information. The frequency of cognitive impairment (CI), defined as at least two BRN-R subtests altered, was 36\%. The figures decreased to $17 \%$ when significant major depression or associated fatigue were excluded. Depressive symptoms were observed in 58\% and anxiety in $76.7 \%$. Conclusions: The results are consistent with those described in the literature. The type of instruments used in the investigation of CC and the definition of CI in MS should be standardized.

(Rev Med Chile 2012; 140: 1437-1444).

Key words: Cognition; Neuropsychology; Multiple sclerosis.

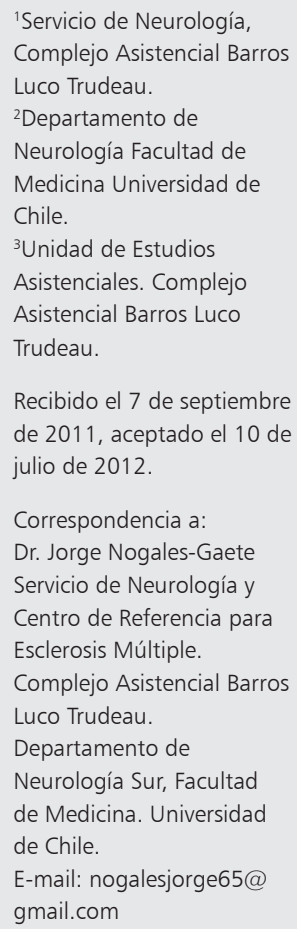

L a esclerosis múltiple (EM) presenta alteraciones cognitivas (AC) y psiquiátricas entre 40 y $60 \%$ de los pacientes, siendo discapacitante en 6 a $10 \%{ }^{1-4}$.

Las AC dependen del tamaño, actividad, localización, número de placas desmielinizantes y severidad de la degeneración axonal ${ }^{4,5}$. Los dominios cognitivos más afectados en test neuropsicológicos son: memoria verbal, velocidad de procesamiento de la información, atención, función ejecutiva, percepción visuespacial y fluencia verbal ${ }^{6}$. Dado que la exploración clínica y pruebas de tamizaje cognitivo como el mini mental test de Folstein (MMES) tienen baja sensibilidad en su pesquisa, se han desarrollado baterías que permiten su identificación precoz y control evolutivo ${ }^{7,8}$. La batería neuropsicológica repetible breve de Rao (BRN$\mathrm{R})$ es el instrumento más usado al respecto ${ }^{3,9,10}$. 
Este tiene 15 formas diferentes, que permiten su aplicación reiterada en el tiempo, a fin de evitar el aprendizaje. Este trabajo presenta los resultados de evaluaciones cognitivas y psicológicas sistemáticas realizadas a 129 pacientes con EM recurrente remitente (EM-RR) atendidos consecutivamente en el programa piloto (PP) nacional de tratamiento con inmunomoduladores del ministerio de salud chileno $^{11}$.

El PP funcionó entre junio de 2008 y junio de 2010, posteriormente la enfermedad ingresó al régimen de patologías con garantías explicitas (GES) de cobertura nacional. En sus aspectos operativos el Servicio de Neurología del Complejo asistencial Barros Luco operó como centro de referencia nacional del PP.

En los primeros seis meses del programa se excluyó a mayores de 50 años y con enfermedad mayor de 4, posteriormente los criterios de ingreso consideraron EM-RR según criterios de Mc Donald $2005^{12}$, con enfermedad activa clínica o imagenológicamente en los dos últimos años y nivel igual o menor a 6,5 en la escala ampliada de discapacidad de Kurztke (EDSS). Al modificarse los criterios se reevaluaron los casos que no calificaron inicialmente.

Investigamos la frecuencia de $\mathrm{AC}$, estimamos el porcentaje de deterioro cognitivo (DC) y comunicamos algunas propiedades psicométricas de la BRN-R.

\section{Material y Método}

Se registraron datos de: presentación, evolución clínica, manejo de la enfermedad, medición de la discapacidad física mediante EDSS y escala funcional compuesta (MSFC), evaluación de fatiga a través de escala de intensidad de fatiga de Krupp e investigación de AC mediante la BRN-R y psiquiátricas por escalas de Hamilton para depresión y ansiedad versión adaptada al castellano por Ramos-Brieva y Cordero ${ }^{13}$.

El análisis de resultados sobre AC y psiquiátricas se realizó por categorías etarias: menores de 35 años, entre 35 y 49 años, y mayores de 49 . El nivel educacional se categorizó según la organización del sistema educacional chileno: 8 o menos años, entre 9 y 12 años y 13 o más años.

Como referencia de normalidad de rendimiento para BRN-R, en ausencia de datos nacionales, usamos valores de autores argentinos y españoles en población hispanoparlante ${ }^{14,15}$. Investigamos la relación de $\mathrm{AC}$ con edad, género, nivel educacional, presencia e intensidad de depresión o ansiedad y grado de EDSS.

En ausencia de una única definición de DC y para estimar su frecuencia lo definimos de manera arbitraria, como la presencia de un resultado igual o inferior a dos desviaciones estándar (DS) en algún sub-test en al menos dos dominios cognitivos.

La BNR-R y cuestionarios de Hamilton se aplicaron al ingreso y a los 6 meses de iniciado el tratamiento inmunomodulador. La BNR-R consiste en un conjunto de 10 sub-test, que conceptualmente miden cuatro dominios cognitivos: memoria verbal, aprendizaje visuespacial, fluencia verbal y velocidad de procesamiento cognitivo. En nuestro trabajo, utilizamos la versión de Cáceres y Vanotti ${ }^{15}$.

Obtuvimos puntajes que estandarizamos a una distribución $\mathrm{Z}$ posteriormente promediamos los puntajes de cada sub-test obteniendo valores para cada dominio. Finalmente, los puntajes por dominios fueron promediados resultando un puntaje $\mathrm{Z}$ total del instrumento.

Investigamos el comportamiento del instrumento en términos de validez y confiabilidad mediante:

A) Validez de constructo: grado en que los resultados se relacionan a constructos psicológicos subyacentes de acuerdo con hipótesis derivadas teóricamente y que conciernen a los conceptos medidos. Se investigó mediante análisis factorial exploratorio (AFE), método factor de componente principal y rotación varimax.

B) Validez de criterio: se refiere a la validez del instrumento comparada con algún criterio externo. Se evaluó midiendo la asociación entre los resultados del puntaje $\mathrm{Z}$ total y por dominio con variables relacionadas a la función cognitiva utilizando regresión lineal univariada. Se reportan los intervalos de confianza de 95\%. Las exploraciones de factores confundentes se realizó mediante análisis multivariado.

C) Confiabilidad: se evaluó mediante el cálculo del coeficiente alfa de Cronbach, el cual cuantifica la consistencia interna de los ítems en cada escala y el instrumento como conjunto. Entrega un valor que nos indica el grado en que los ítems de una escala se relacionan entre si. Valores mayores a 0,7 se consideran aceptables. 


\section{Resultados}

\section{Descripción general}

Evaluamos 129 pacientes, 69\% fueron mujeres. La edad promedio al momento de la evaluación fue 33,4 años con DS de 9,5 y rango entre 12 y 60 años.

Según rango etario se distribuyeron como sigue: 71 pacientes (55\%) tenían menos de 35 años, 53 (41\%) tenían entre 35 y 49, y 5 (3,9\%) más de 49 años. La edad promedio de inicio de la enfermedad fue 29,2 años con una DS de 8,9 una moda 28 años y una mediana 30 . Observamos casos entre 6 y 54 años.

El nivel educacional mostró: 7 pacientes $(5,4 \%)$ con menos de 8 años de escolaridad, 49 (38\%) entre 8 y 12 años, y $73(56,6 \%)$ con 13 o más años. Así, más del 94\% de los pacientes tenían ocho o más años de escolaridad. El nivel de EDSS promedio fue 2,83 (mínimo de 1, máximo de 6,5) y una DS de 1,44. Hubo 48 pacientes con EDSS de 3 o menos y 81 con valor de 3,5 o más. El promedio de fatiga basal fue de 4,2. Fatiga significativa entendida como resultado superior a 5,1 estaba presente en 41 pacientes (32\%).

\section{Ansiedad y depresión}

Observamos la presencia de depresión en $58,1 \%$ y de ansiedad en $76,7 \%$. Depresión severa o más que severa estuvo presente en 12 pacientes, lo que representa 12,3\% de los casos. Ansiedad severa o más que severa la observamos en 6 pacientes.

\section{Evaluación cognitiva}

La Tabla 1 muestra los resultados en cada sub-test de la BNR-R comparándolos, con los promedios obtenidos en controles sanos del estudio RECONEM realizado en controles argentinos ${ }^{14}$. Los resultados promedio resultaron inferiores a los promedios de dichos controles en todos los sub-test del instrumento. Al comparar el rendimiento de nuestros pacientes también con estos controles usando como punto de corte de AC un valor inferior a 2 DS obtuvimos porcentajes que fluctuaron entre 15,5 y $54,3 \%$. El mayor compromiso lo observamos en los dominios memoria verbal y atención sostenida. La frecuencia de AC en al menos un sub-test de la BNR-R, considerando para su definición el mismo criterio antes explicitado fue $62 \%$.

Tabla 1. Resultados de la BNR-R en el grupo evaluado y comparación de los resultados con controles sanos de estudio RECONEM

\begin{tabular}{|c|c|c|c|c|c|c|c|}
\hline $\begin{array}{l}\text { Batería neuropsicológica } \\
\text { repetible breve de Rao }\end{array}$ & $\begin{array}{l}\text { Pro- } \\
\text { medio }\end{array}$ & $\begin{array}{l}\text { Media- } \\
\text { na }\end{array}$ & $\begin{array}{l}\text { Desviación } \\
\text { estándar }\end{array}$ & $\begin{array}{l}\text { Puntaje } \\
\text { mínimo }\end{array}$ & $\begin{array}{l}\text { Puntaje } \\
\text { máximo }\end{array}$ & $\begin{array}{l}\text { \% Bajo } \\
\text { punto } \\
\text { de corte } \\
\text { de } 2 \mathrm{DS}\end{array}$ & $\begin{array}{l}\text { Promedio } \\
\text { controles } \\
\text { sanos estudio } \\
\text { RECONEM }\end{array}$ \\
\hline $\begin{array}{l}\text { Test selectivo de memoria: memoria } \\
\text { largo plazo-almacenamiento }\end{array}$ & 34,1 & 34,5 & 14,33 & 0 & 66 & $44,25 \%$ & 51,7 \\
\hline $\begin{array}{l}\text { Test selectivo de memoria: memoria } \\
\text { largo plazo-recuperación }\end{array}$ & 23,7 & 23 & 14,51 & 0 & 66 & $33,3 \%$ & 42 \\
\hline $\begin{array}{l}\text { Test selectivo de memoria: memoria } \\
\text { diferida }\end{array}$ & 6,2 & 6 & 2,68 & 0 & 12 & $54,3 \%$ & 9,9 \\
\hline Test $7 / 24$ respuestas correctas & 25,4 & 27 & 7,25 & 7 & 35 & $28,7 \%$ & 30,7 \\
\hline Test $7 / 24$ recuerdo inmediato & 4,8 & 5 & 2,32 & 0 & 7 & $20,2 \%$ & 5,9 \\
\hline Test $7 / 24$ recuerdo diferido & 6 & 5 & 2,16 & 0 & 7 & $17,8 \%$ & 5,8 \\
\hline $\begin{array}{l}\text { Fluencia verbal: versión } \\
\text { fonológica }\end{array}$ & 32,6 & 30 & 13,18 & 0 & 73 & $20,2 \%$ & 42,1 \\
\hline Test símbolo digito & 41 & 41 & 13,68 & 1 & 70 & 37,55 & 47,3 \\
\hline Passat Versión 3 s. & 31 & 30 & 14,39 & 0 & 59 & $15,5 \%$ & 38,9 \\
\hline Passat Versión 2 s. & 23,7 & 23 & 11,41 & 0 & 58 & $44,5 \%$ & 50,1 \\
\hline
\end{tabular}


Estimamos el porcentaje de DC, utilizando la definición antes señalada, mediante la comparación de su rendimiento con otros controles ${ }^{15}$ en quienes los resultados se ajustaron por edad y nivel educacional de modo parecido al nuestro. Con la definición adoptada obtuvimos 46 pacientes que presentaban resultados inferiores a 2 DS en algún sub-test en al menos dos dominios cognitivos esto es 36\%.

Para aislar factores de confusión, excluimos quienes presentaban depresión mayor y/o fatiga significativa; de esta forma el grupo se redujo a 22 pacientes (17\%). Así nuestra serie presentó un rango de DC que oscilaba entre $17 \%$ y $36 \%$. En el subgrupo de pacientes con DC el valor promedio EDSS fue de 3,5 versus 2,6 en el subgrupo sin DC, ello resultó estadísticamente significativo (p: 0,05).

De los 129 pacientes evaluados sólo 5 presentaban sospecha de disfunción cognitiva en la evaluación clínica habitual (historia o MMES). En todos se pesquisó alguna $\mathrm{AC}$ y en tres de ellos se configuró DC según nuestra definición. En sólo 2 de los 22 pacientes con DC (sin depresión o fatiga significativa) el MMES estaba alterado. En 98 pacientes se realizó una segunda aplicación de la BNR-R a los 6 meses de la inicial esta mostró mantención de $\mathrm{DC}$ en 10 y persistencia de $\mathrm{AC}$ en todos los pacientes con DC inicial. Por otro lado, $47 \%$ de quienes no presentaron DC en la evaluación basal, si lo mostraron en el control.

El perfil de AC encontradas se resume como sigue (Tablas 2, 3 y 4 ):

1) Dominios cognitivos más afectados de forma aislada: memoria verbal, velocidad de procesamiento de la información y fluencia verbal con 47,36 y $21 \%$ respectivamente.

2) Combinaciones de compromisos más frecuentes:

- Memoria verbal y velocidad de procesamiento de la información 30 casos $(23,2 \%)$.

- Fluidez verbal y velocidad de procesamiento de la información: 20 casos $(15,5 \%)$.

- Memoria verbal y fluidez verbal también 20 casos $(15,5 \%)$.

\section{Propiedades de la BRN-R}

\subsection{Validez de constructo}

Validez de constructo: La solución significativa del AFE se logró con tres factores, con 78,7\% de la varianza del instrumento (Kaiser-Meyer-Olkin $0,78)$ y todos los ítems con unicidades inferiores
Tabla 2. Porcentaje de AC aislada por dominio cognitivo en los pacientes evaluados*

$\begin{array}{ll}\text { A. Memoria verbal } & 43,4 \% \text { (56 casos) } \\ \text { B. Velocidad de procesamiento } & 36,4 \% \text { (47 casos) } \\ \text { C. Fluidez verbal } & 20,9 \% \text { (27 casos) } \\ \text { D. Aprendizaje visuespacial } & 18,6 \% \text { (24 casos) }\end{array}$

* Se consideró punto de corte valor inferior al percentil 2DS\% de estudio RECONEM. Se excluyeron pacientes con depresión y fatiga severa.

Tabla 3. Número de dominios cognitivos alterados en los pacientes evaluados*

\begin{tabular}{|lll|}
\hline A. 1 dominio cognitivo alterado & $26,3 \%$ (34 casos) \\
\hline B. 2 dominios cognitivos alterados & $17,8 \%$ (23 casos) \\
\hline C. 3 dominios cognitivos alterados & $13,1 \%$ (17 casos) \\
\hline D. $\quad 4$ dominios cognitivos alterados & $4,6 \% \quad$ (6 casos) \\
\hline
\end{tabular}

* Se consideró punto de corte valor inferior al percentil 2DS\% de estudio RECONEM. Se excluyeron pacientes con depresión y fatiga severa.

Tabla 4. Combinaciones de afectación de dominios cognitivos en los pacientes evaluados*

\begin{tabular}{|c|c|}
\hline $\begin{array}{l}\text { - Memoria verbal + velocidad } \\
\text { de procesamiento }\end{array}$ & $23,25 \%$ (30 casos) \\
\hline - Memoria verbal + fluidez verbal & $15,50 \%$ (20 casos) \\
\hline $\begin{array}{l}\text { - Memoria verbal + aprendizaje } \\
\text { visuespacial }\end{array}$ & $12,40 \%$ ( 16 casos) \\
\hline $\begin{array}{l}\text { - Aprendizaje visuespacial + } \\
\text { velocidad de procesamiento }\end{array}$ & $10,07 \%$ ( 13 casos) \\
\hline $\begin{array}{l}\text { - Aprendizaje visuespacial + } \\
\text { fluidez verbal }\end{array}$ & $5,42 \%$ (7 casos) \\
\hline $\begin{array}{l}\text { - Fluidez verbal + velocidad } \\
\text { de procesamiento }\end{array}$ & $15,50 \%$ (20 casos) \\
\hline
\end{tabular}

*Se consideró punto de corte valor inferior al percentil 2DS\% de estudio RECONEM. Se excluyeron pacientes con depresión y fatiga severa.

a 50\%. Ello demuestra que este se comportó de manera válida en el grupo estudiado.

\subsection{Validez de criterio}

La regresión lineal univariada evidenció que el puntaje Z total de la BRN-R se relacionó de manera significativa con escolaridad (coeficiente para $\geq 13$ años de estudios 0,87 [IC 95\% 0,37 a 1,36]), 
puntaje de depresión de Hamilton (coeficiente: (-) 0,03 (IC 95\% (-) 0,05 a (-) 0,01) y EDSS basal (coeficiente: (-) 0,19 [IC 95\% (-) 0,27 a (-) 0,12]). No evidenciamos relación significativa con edad ni con ansiedad. En análisis multivariado persistió relación significativa entre $\mathrm{Z}$ total de la $\mathrm{BRN}-\mathrm{R}$ con escolaridad y puntaje EDSS.

\subsection{Confiabilidad}

Coeficiente alfa de Cronbach: el instrumento total y las sub-escalas de memoria verbal y velocidad de procesamiento de la información presentaron un alfa de Cronbach igual o superior a 0,7 lo que demuestra confiabilidad adecuada. El menor valor lo observamos para la sub-escala de aprendizaje visuespacial que presento un resultado de 0,50 [IC $95 \% 0,30-0,65$ ].

\section{Discusión}

Las características demográficas referidas a género, edad de presentación de la enfermedad, edad al momento de la evaluación y nivel educacional del grupo de pacientes evaluados son similares a lo comunicado en otras series de pacientes latinoamericanos ${ }^{13-17}$. En términos de sus propiedades psicométricas, la $\mathrm{BRN}-\mathrm{R}$ se mostró como un instrumento válido y confiable.

Nuestros pacientes presentan discapacidad física más bien leve a moderada concentrándose los casos con puntajes en torno al escalón 3 de la escala EDSS. Por tratarse nuestro programa de una actividad de práctica clínica habitual, no conformamos un grupo control con quienes comparar los resultados obtenidos en la BNR-R, por ello ocupamos como referencia de normalidad los datos publicados de los estudios RECONEM y de Sepulcre y cols en población hispanoparlante ${ }^{14,15}$. Así, en todos los sub-test de la BRN-R los resultados promedio de nuestros pacientes fueron inferiores a dichos controles tanto al analizar la información de forma aislada como ajustada por nivel de educacional y edad resultando en $62 \%$ la frecuencia de alguna AC cifra dentro de lo comunicado en la literatura ${ }^{3,14,15}$. Los dominios afectados con más frecuencia tanto de forma aislada como en combinación fueron memoria verbal y velocidad de procesamiento de la información, donde los porcentajes bajo el promedio fueron 54 y $44,5 \%$ respectivamente en nuestros pacientes.
Lo anterior se reitera al investigar la magnitud del compromiso para cada sub-test de la BNR-R usando el criterio de 2 DS como punto de corte de normalidad. Estos datos están en concordancia con la literatura respecto de la presencia de AC desde etapas iniciales de la enfermedad en pacientes con poca discapacidad física ${ }^{18-21}$.

Otro hecho señalado en la literatura y que observamos en nuestros pacientes es el compromiso de lenguaje en términos alteración en la generación de palabras, lo que contrasta con la ausencia de otras alteraciones como la ocurrencia de afasias evaluada mediante instrumentos como el test de Boston ${ }^{3,35}$.

Consignamos algunas diferencias, metodologicas, de nuestro estudio en relación con nuestras principales referencias de comparación:

1) En el estudio de Sepulcre y cols ${ }^{15}$ la versión de la BNR-R difiere en las pruebas de aprendizaje visuespacial y fluencia verbal de las que aplicamos nosotros (10/36 vs $7 / 24$ y test de fluencia verbal semántico vs fonémico).

2) El estudio RECOMEM uso percentil 5 como punto de corte de AC e incluyo pacientes con diferentes formas evolutivas de EM y en el nuestro sólo pacientes con EM-RR.

En relación con la definición de DC en EM, pese a que existe amplia información en la literatura sobre descripción de AC, no existe consenso sobre la definición de DC ya sea para investigación o práctica clínica. Lo anterior incluye el instrumento de medición utilizado, cantidad y/o tipo de dominios cognitivos alterados y la magnitud de dicha alteración.

A modo de ejemplo: Deloire $\mathrm{M}$ y cols ${ }^{22}$ investigan la presencia de DC utilizando como definición la presencia, en un paciente, de un resultado inferior al percentil 5 en dos test de una batería de pruebas que incluía la BNR-R más el Go/NoGoStroop test. Con ello obtienen un resultado de un 47,8\% de DC en 58 pacientes con EM-RR.

En la misma línea, en la literatura se señalan diversas definiciones de DC usando como instrumento de medición la BNR-R. Ellas se fundamentan en la existencia de alteración en 2, 3, 5 ó más subtest de la misma con rendimiento bajo 2 DS de un control de referencia como punto de corte ${ }^{22-24}$.

Con estas consideraciones, optamos por una definición que incluyera el compromiso de dos dominios con una magnitud superior a 2 DS de 
una población de referencia, en este caso la estudiada por Sepulcre y cols en población argentina, en quienes la edad y escolaridad se ajustó de modo similar al nuestro ${ }^{15}$. De esta forma, el porcentaje encontrado fue primero de $35 \%$, reduciéndose a $17 \%$ al excluir los pacientes con depresión mayor y fatiga significativa por considerarlos eventuales factores de confusión para el rendimiento. Este resultado es parecido a lo comunicado en resultados basales del estudio "Cognitive Impairment in Multiple Sclerosis study goup" donde se evaluaron 550 pacientes con EM-RR y cuya definición de DC considero la alteración de 3 o más sub-test de la BNR-R ${ }^{23}$.

Respecto del perfil de la afectación cognitiva de nuestros pacientes, observamos también lo referido por otros autores en cuanto a mayor compromiso de la memoria verbal, velocidad de procesamiento de la información y fluencia verbal $^{22-27}$.

En el grupo de pacientes evaluados sólo 5 presentaban sospecha de disfunción cognitiva en la evaluación clínica habitual (historia o MMES). En todos ellos se pesquisó alguna AC y en tres de ellos se constituyó DC según nuestra definición. Además, en sólo 2 de 22 pacientes con DC el MMES estaba alterado. Deseamos precisar que estamos hablando de alteraciones en test cognitivos cuya relevancia en la vida diaria de nuestros pacientes por ahora no es clara.

En relación con los síntomas psiquiátricos, encontramos presencia de sintomatología depresiva en $58 \%$ de los pacientes. La mayoría, sin embargo, fue de intensidad leve o moderada siendo sólo en $12 \%$ de los casos de una severidad tal como para calificar el episodio de depresivo mayor. En todos estos pacientes la evaluación clínica del psiquiatra confirmó el diagnóstico. Estos resultados son comparables a lo comunicado en la literatura que señala una frecuencia de depresión mayor en torno al $20 \%$ en EM, aunque la comunicación de síntomas depresivos puede superar el 50\% como ocurrió en nuestra serie ${ }^{28,29}$.

En este aspecto es preciso puntualizar que el instrumento usado por nosotros en la investigación de síntomas depresivos (cuestionario de Hamilton) no es el que predomina en la mayoría de las publicaciones sobre el tema en que se señala mayoritariamente el uso del inventario de Beck y la escala hospitalaria de depresión ${ }^{30-33}$. No obstante ello, nos pareció útil su uso debido a que constituye un instrumento aplicable a población ambulatoria consistente en una evaluación externa de los síntomas, que permite observar la evolutividad del cuadro y que brinda al entrevistador la posibilidad de distinguir las quejas somáticas debidas a un cuadro depresivo y las originadas por una afección médica.

En resumen el grupo de pacientes evaluados presenta características demográficas, clínicas y neuropsiquiatricas similares a lo comunicado en la literatura. La cantidad de AC y DC encontrados en nuestros casos ratifica una inquietud no resuelta cual es, como señalamos antes, la correlación de dichas alteraciones, tanto en sus diferentes grados de severidad como en los dominios afectados, con el eventual impacto en la vida diaria del paciente. Por otro lado, es necesaria la obtención de cifras de referencia nacionales para valores de normalidad de la BNR-R y por supuesto la evaluación sus propiedades psicométricas. Esto constituye una necesidad no sólo de investigación sino fundamentalmente clínica debido a las implicancias terapéuticas a futuro ${ }^{34}$.

También creemos que es de gran importancia mantener el seguimiento de los pacientes en cuanto a la evolución de su rendimiento cognitivo y su relación con otras variables clínicas incluida calidad de vida. Lo anterior es importante independiente que sean consideradas como desenlace de respuesta a tratamiento con inmunomoduladores, en cuanto permiten planificar una atención más integral ${ }^{34-37}$.

\section{Referencias}

1. Compston A, Coles A. Multiple Sclerosis. Lancet 2002; 359 (9313): 1221-31.

2. Rao SM, Leo GJ, Ellington L, Nauertz T, Bernardin L, Unverzagt F. Cognitive dysfunction in multiple sclerosis II. Impact on employment and social functioning. Neurology 1991; 41 (5): 692-6.

3. Prakash RS, Snook EM, Lewis JM, Motl RW, Kramer AF. Cognitive impairments in relapsing-remitting multiple sclerosis: a meta-analysis. Mult Scler 2008; 14 (9): 1250-61.

4. Rao SM. Neuropsychology of multiple sclerosis. Curr Opin Neurol 1995; 8 (3): 216-20.

5. Jongen PJ, Ter Horst AT, Brands AM. Cognitive impairment in multiple sclerosis. Minerva Med 2012; 103 (2): 73-96.

6. Rodrigues DN, Paes RA, Vasconselos CC, Landeira- 
Fernández J, Alvarenga MP. Different cognitive profiles of Brazilian patients with relapsing-remitting and primary progressive mukltiple sclerosis. Arq Neuropsiquiatry 2011; 69 (4): 590-5.

7. Beatty WW, Goodkin DE. Screening for cognitive impairment in multiple sclerosis. An evaluation of the Mini-Mental State Examination. Arch Neurol 1990; 47 (3): 297-301.

8. Rao SM, Leo GJ, Bernardin L, Unverzagt F. Cognitive dysfunction in multiple sclerosis. I. Frequency, patterns, and prediction. Neurology 1991; 41 (5): 685-91.

9. Boringa JB, Lazeron RH, Reuling IE, Adèr HJ, Pfennings L, Lindeboom J, et al. The brief repeatable battery of neuropsychological tests: normative values allow application in multiple sclerosis clinical practice. Mult Scler 2001; 7 (4): 263-7.

10. Achiron A, Barak Y. Cognitive impairment in probable multiple sclerosis. J Neurol Neurosurg Psychiatry 2003; 74 (4): 443-6.

11. Nogales-Gaete J, Aracena R, Agurto P, Cepeda S, Figueroa $\mathrm{C}$, González $\mathrm{C}$, et al. Programa piloto para pacientes beneficiarios de FONASA, que padecen Esclerosis Múltiple. Tratamiento con Inmunomoduladores en el Sistema Público de Salud de Chile. Informe del primer año, 10 de julio 2008-30 de junio 2009. Rev Chil NeuroPsiquiat 2010; 48: 9-92.

12. Polman $\mathrm{CH}$, Reingold SC, Edan G, Filippi M, Hartung HP, Kappos L, et al. Diagnostic criteria for multiple sclerosis: 2005 revisions to the "McDonald Criteria". Ann Neurol 2005; 58 (6): 840-6.

13. Ramos-Brieva JA, Cordero Villafáfila A. En Hernández R, Sandoval G, Cáceres F, Garcea O, Villoslada P. Validación de la versión castellana de la escala de Hamilton para la depresión. Actas Luso-Esp Neurol Psiquiatr 1986; 14: 324-34.

14. Cáceres F, Vanotti S, Rao S; RECONEM Workgroup. Epidemiological characteristics of cognitive impairment of multiple sclerosis patients in a Latin American country. J Clin Exp Neuropsychol 2011; 33 (10): 1094-8. Epub 2011 Oct 6.

15. Sepulcre J, Vanotti S, Hernández R, Sandoval G, Cáceres F, Garcea $\mathrm{O}$, et al. Cognitive impairment in patients with multiple sclerosis using the Brief Repeatable Battery-Neuropsychology test. Mult Scler 2006; 12 (2): 187-95.

16. Vizcarra-Escobar D, Cava-Prado L, Tipismana-Barbarán M. Esclerosis múltiple en Perú. Descripción clinicoepidemiológica de una serie de pacientes. Rev Neurol 2005; 41 (10): 591-5.

17. Cardoso E, Fukuda T, Pereira J, Seixas J, Miranda R, Rodrigues B, et al. Clinical and epidemiological profile of multiple sclerosis in a reference center in the State of Bahia, Brazil. Arq Neuropsiquiatr 2006; 64 (3B): 727-30.

18. Fragoso YD, Fiore AP. Description and characteristics of 81 patients attending the Reference Center for Multiple Sclerosis of the coastal region of the state of São Paulo-Brazil. Arq Neuropsiquiatr 2005; 63: 741-4.

19. Nieto Barco A, Sánchez López M, Barroso Ribal J, Olivares Pérez T, Hernández Pérez MA. Alteraciones cognitivas en etapas iniciales de la esclerosis múltiple y su relación con el estado de ánimo, variables demográficas y clínicas. Psicothema 2008; 20 (4): 583-8.

20. Dujardin K, Sockeel P, Cabaret M, De Sèze J, Vermersch P. [BCcogSEP: a French test battery evaluating cognitive functions in multiple sclerosis]. Rev Neurol (Paris) 2004; 160 (1): 51-62.

21. Siepman TA, Janssens AC, de Koning I, Polman CH, Boringa JB, Hintzen RQ. The role of disability and depression in cognitive functioning within 2 years after multiple sclerosis diagnosis. J Neurol 2008; 255 (6): 910-6.

22. Deloire M, Ruet A, Hamel D, Bonnet M, Brochet B. Early cognitive impairment in multiple sclerosis predicts disability outcome several years later. Mult Scler 2010; 16 (5): 581-7.

23. Patti F, Amato MP, Trojano M, Bastianello S, Tola MR, Goretti B, et al; COGIMUS Study Group. Cognitive impairment and its relation with disease measure in mildly disabled patients with relapsing-remitting multiple sclerosis: baseline results from the Cognitive Impairment in Multiple Sclerosis (COGIMUS) study. Mult Scler 2009; 15 (7): 779-88.

24. Amato MP, Portaccio E, Goretti B, Zipoli V, Ricchiuti L, De Caro MF, et al. The Rao's Brief Repeatable Battery and Stroop Test: normative values with age, education and gender corrections in an Italian population. Mult Scler 2006; 12 (6):787-93.

25. Portaccio E, Goretti B, Zipoli V, Siracusa G, Sorbi S, Amato MP. A short version of Rao's Brief Repeatable Battery as a screening tool for cognitive impairment in multiple sclerosis. Clin Neuropsychol 2009; 23 (2): 26875.

26. Comi G, Filippi M, Martinelli V, Campi A, Rodegher M, Alberoni M, et al. Brain MRI correlates of cognitive impairment in primary and secondary progressive multiple sclerosis J Neurol Sci 1995; 132 (2): 222-7.

27. Camp SJ, Stevenson VL, Thompson AJ, Miller DH, Borras C, Auriacombe S, et al. Cognitive function in primary progressive and transitional progressive multiple sclerosis: a controlled study with MRI correlates. Brain 1999; 122 (7): 1341-1348.

28. Amato MP, Zipoli V, Goretti B, Portaccio E, De Caro 
MF, Ricchiuti L, et al. Benign multiple sclerosis: cognitive, psychological and social aspects in a clinical cohort. J Neurol 2006; 253 (8): 1054-9.

29. Siegert RJ, Abernethy DA. Depression in multiple sclerosis: a review. J Neurol Neurosurg Psychiatry 2005; 76 (4): 469-75.

30. Beiske AG, Svensson E, Sandanger I, Czujko B, Pedersen ED, Aarseth JH, et al. Depression and anxiety amongst multiple sclerosis patients. Eur J Neurol 2008; 15 (3): 239-45.

31. Da Silva AM, Vilhena E, Lopes A, Santos E, Gonçalves MA, Pinto C, et al. Depression and anxiety in a Portuguese MS population: Associations with physical disability and severity of disease. J Neurol Sci 2011; 306 (1-2): 66-70.

32. Suh Y, Motl RW, Mohr DC. Physical activity, disability, and mood in the early stage of multiple sclerosis. Disabil Health J 2010; 3 (2): 93-98.
33. Baumstarck-Barrau K, Simeoni MC, Reuter F, Klemina I, Aghababian V, Pelletier J, et al. Cognitive function and quality of life in multiple sclerosis patients: a crosssectional study. BMC Neurol 2011; 2: 11-17.

34. Siniscalchi A, Gallelli L, Tolotta GA, Loiacono D, De Sarro G. Open, uncontrolled, nonrandomized, 9-month, off-label use of bupropion to treat fatigue in a single patient with multiple sclerosis. Clin Ther 2010; 32 (12): 2030-2034.

35. Patti F. Cognitive impairment in multiple sclerosis. Mult Scler 2009; 15 (1): 2-8.

36. DeSousa EA, Albert RH, Kalman B. Cognitive impairments in multiple sclerosis: a review. Am J Alzheimers Dis Other Demen 2002; 17 (1): 23-9.

37. Patti F, Leone C, D'Amico E. Treatment options of cognitive impairment in multiple sclerosis. Neurol Sci 2010; 31: S265-9. 